\title{
Particle Emission-Dependent Timing Noise of Pulsars?
}

\author{
LIU Xiong-Wei, NA Xue-Sen, XU Ren-Xin, QIAO Guo-Jun \\ School of Physics and State Key Laboratory of Nuclear Physics and Technology, \\ Peking University, Beijing 100871, China; \\ xiongwliu@163.com
}

\begin{abstract}
Though pulsars spin regularly, the differences between the observed and predicted ToA (time of arrival), known as "timing noise", can still reach a few milliseconds or more. We try to understand the noise in this study. As proposed by $\mathrm{Xu}$ and Qiao in 2001, both dipole radiation and particle emission would result in pulsar braking. Accordingly, possible fluctuation of particle current flow is suggested here to contribute significant ToA variation of pulsars. We find that the particle emission fluctuation could lead to timing noise which cannot be eliminated in timing process and that a longer period fluctuation would arouse a stronger noise. The simulated timing noise profile and amplitude are in agreement with the observed timing behaviors on the timescale of years.
\end{abstract}

PACS: 97.60.Gb, 05.40.Ca

Why do pulsars spin down? This is a question still not fully answered even more than 40 years later since the discovery of the first pulsar. It is generally suggested that pulsars spin down via magneto-dipole radiation, by which the ages and the surface magnetic fields are estimated accordingly. However, it was proposed by Xu and Qiao (2001) that both dipole radiation and relativistic particle emission powered by a unipolar generator can result in the loss of pulsar rotation energy, and the observed braking indices $(<3)$ could be understood then. ${ }^{[1,2]}$ This opinion is consistent with later simulation ${ }^{[3]}$ and observation. ${ }^{[4]}$ In this Letter, we focus on further implication of the braking mechanism to timing behavior in Xu and Qiao's model.

Timing noise is the residual of pulsar time of arrival (ToA) after fitted by the timing model. It reflects the effects of unknown elements to ToA. A lot of models were proposed to explain timing noise, such as the random walk in pulse frequency, ${ }^{[5]}$ the free-precession of neutron star, ${ }^{[6]}$ the unmodelled companions, ${ }^{[7,8]}$ and the effect of gravitational waves. ${ }^{[9]}$ However, the noise still cannot be eliminated completely, especially in long timescale of years. ${ }^{[10-13]}$ On the other hand, the pulsar flux density monitoring of the Green Bank ${ }^{[14]}$ indicates that the pulsar emission may not be absolutely stable. It is then reasonable in Xu and Qiao's model that there exits fluctuation in the relativistic particle emission, which would consequently contribute to the timing noise. We will take the fluctuation in pulsar emission into account in timing process, in this work, and try to find the relationship between the fluctuation and timing noise. 
The rotational energy loss rate is

$$
-I \Omega \dot{\Omega}=\dot{E}=\dot{E}_{d}+\dot{E}_{u}
$$

where $I$ is the moment of inertia of a pulsar, $\Omega$ and $\dot{\Omega}$ are its angular velocity and the first derivative, $\dot{E}$ is the loss rate of rotational energy, and $\dot{E}_{d}$ and $\dot{E}_{u}$ are the powers of dipole radiation and relativistic particle flow, respectively. ${ }^{[1]}$ When there is a fluctuation in $\dot{E}_{u}$, it becomes

$$
\dot{E}_{u}=\dot{\dot{E}}_{u}(1+\delta)
$$

where $\overline{\dot{E}}_{u}$ is the stable value of $\dot{E}_{u}$, and $\delta$ is the fluctuation. For different pulsars the relative quantities of $\dot{E}_{d}$ and $\dot{E}_{u}$ are different because the magnetic inclinations are distinct and maybe the radiation mechanisms are not the same. However, for an individual pulsar these two components are sufficiently decided in a period of time and generally in a same order of magnitude. For the above reasons, and considering the dipole radiation is stable, we take

$$
\dot{E}_{d}=n \times \overline{\dot{E}}_{u},
$$

where $n$ is a constant and decided by the magnetic inclination and radiation mechanism.

From Eqs. (1), (2) and (3) we obtain

$$
-I \Omega \dot{\Omega}=\overline{\dot{E}}_{u}(n+1+\delta) .
$$

Performing integration to both sides it becomes

$$
\frac{1}{2} I\left[\Omega_{0}^{2}-\Omega(T)^{2}\right]=\overline{\dot{E}}_{u}\left[(n+1) T+\int_{0}^{T} \delta(t) d t\right]
$$

where $\Omega_{0}$ is the value of $\Omega$ at the beginning time, and we suppose that the moment of inertia $I$ is constant because it changes sufficiently small. When there is no fluctuation in $\dot{E}_{u}$, Eq. (5) becomes

$$
\frac{1}{2} I\left[\Omega_{0}^{2}-\Omega^{\prime}(T)^{2}\right]=\overline{\dot{E}}_{u}(n+1) T,
$$

where $\Omega^{\prime}(T)$ is the expected value when fluctuation is zero. Equation (5) minus Eq. (6) is

$$
\frac{1}{2} I\left[\Omega^{\prime}(T)^{2}-\Omega(T)^{2}\right]=\overline{\dot{E}}_{u} \int_{0}^{T} \delta(t) d t .
$$

Considering the spin of pulsar changes very slowly, we obtain

$$
\Omega^{\prime}(T)-\Omega(T)=\frac{\overline{\dot{E}}_{u}}{I \Omega_{0}} \int_{0}^{T} \delta(t) d t .
$$

From Eqs. (4) and (8) we have

$$
\Omega^{\prime}(T)-\Omega(T)=\frac{-\dot{\Omega}_{0}}{n+1+\delta_{0}} \int_{0}^{T} \delta(t) d t .
$$


Performing integration to both sides we obtain

$$
\frac{-\dot{\Omega}_{0}}{n+1+\delta_{0}} \int_{0}^{\tau} \int_{0}^{T} \delta(t) d t d T=\int_{0}^{\tau}\left[\Omega^{\prime}(T)-\Omega(T)\right] d T=\Phi^{\prime}(\tau)-\Phi(\tau)=-\Delta \Phi(\tau)=-\Omega_{0} R,
$$

where $\Phi$ is the phase of the pulsar, and $R$ is a provisional timing residual. Thus one has

$$
R=\frac{\dot{\Omega}_{0}}{\left(n+1+\delta_{0}\right) \Omega_{0}} \int_{0}^{\tau} \int_{0}^{T} \delta(t) d t d T=-\frac{\dot{P}_{0}}{\left(n+1+\delta_{0}\right) P_{0}} \int_{0}^{\tau} \int_{0}^{T} \delta(t) d t d T,
$$

$P_{0}$ and $\dot{P}_{0}$ are the period and its first derivative at beginning time. Equation (11) reflects the relationship between the fluctuation and timing residual. We can obtain the real timing residual $\Re$ by performing least-squares-fitting to $R$.

To understand more clearly about Eq. (11), we try to provide a simple example. Let $\delta(t)=$ $a \sin \left(2 \pi t / t_{0}\right)$, one has

$$
\Re \cong \frac{a \dot{P}_{0} t_{0}^{2}}{4 \pi^{2}\left(n+1+\delta_{0}\right) P_{0}} \sin \left(2 \pi \frac{t}{t_{0}}\right) .
$$

From Eq. (12) we can see that longer timescale variation will cause stronger noise because $\Re \propto t_{0}{ }^{2}$. For a normal pulsar with $P_{0}=0.1 \mathrm{~s}$ and $\dot{P}_{0}=1 \times 10^{-14}$, when $a=0.01, t_{0}=y \times 3.15 \times 10^{7} \mathrm{~s}$ and $n=1$, we obtain $\Re \cong 0.013 \times y^{2} \times \sin \left(2 \pi t / t_{0}\right)$ s. It is a very strong noise at the timescale of years.

We further do a simulation with Eq. (11). Three sets of random data with different Hurst parameter $H$, which reflects the time dependence of a time series data, ${ }^{[15]}$ are produced to simulate three types of irregular fluctuations in $\dot{E}_{u}$. As is shown in Fig. 1, each set of data has 10000 points. The first set has more short period components, with $H=0.4$; the second set is approximate white noise, with $H=0.6$; the third one has more long period components, with $H=0.8$. In this simulation we take $\left(n+1+\delta_{0}\right) P_{0}=0.1 \mathrm{~s}$ and $\dot{P}_{0}=1 \times 10^{-14}$. The corresponding timing noises are shown in Fig. 2. The figures indicate that if the particle emission has a random variation with extent of about $1 \%$ in daily timescale, the flux density from the most distant pulsars varies less than $5 \%,{ }^{[14]}$ it will lead to a timing noise with range of dozens of millisecond in 2000 days (shown on the left of Fig. 2), and several hundreds of millisecond in 10000 days (shown on the right of Fig. 2 ). These curves also show the fluctuation with more long period components to cause stronger noise, which accords with Eq. (12) very well.

Compared Fig. 2 with the observations, Fig. 1 in Ref. [11] and Figs. 1 and 2 in Ref. [12], we find that they have some common features. (1) The majority time curves have about one period-like main structure no matter how long the time spans are (see Refs. [10,13] for more examples), so that one cannot distinguish which one has the long or short time span, just depend on their profiles, even for the same pulsar. (2) The range from the minimum to maximum residual with longer time span is larger than the one with shorter time span for each pulsar, which is in agreement with Eq. (12). (3) The time curve of the shorter time span is extremely similar to the corresponding time span part of the longer one, which is natural because of the integral relation in Eq. (11). 
Recently, Lyne et al. ${ }^{[16]}$ proposed another idea of producing timing noise to pulsar, namely variations of the pulsar spin-down states variations lead to timing noise. This phenomenologically explains the origin of some quasi-periodic structures, which lie on lower-frequency structures of some timing noise. However, it cannot give rise to the ubiquitous lower-frequency structures in long time scales, which are what we try to do in this study.

The statistics results from most pulsar timing noises are in agreement with our model. Soon after we put our work on arXiv, a statistics from Ryan et al. gives $\sigma_{T N, 2} \propto \nu^{0.9 \pm 0.2}|\dot{\nu}|^{1.0 \pm 0.05},[17]$ which is consistent with Eq. (11) very well. From observations, Cordes and Downs, ${ }^{[18]}$ D'Alessandro et al. ${ }^{[10]}$ and Ryan et al. ${ }^{[17]}$ all suggested that a mixture of random walks in $\nu$ and $\dot{\nu}$ is compatible with the timing noise, whereas we propose here a natural physical origin as shown in Eq. (4). We can have the timing noises of millisecond pulsar and AXP to be orders of $10^{2}$ ns and $10 \mathrm{~s}$, respectively, from Eq. (11), which are consistent with the observations.

In summary, our model shows that the fluctuation of particle emission will cause significant timing noise. We emphasize that there could be other kinds of the fluctuation (e.g., $\delta$ ), nevertheless the long period composition of variation contributes larger to the noise. The simulation accords with long (years) timescale noises both in range and profile features. Simultaneously, our work supports the opinion that the pulsar emission is not always stable, which is important to the research of pulsar radiation and the understanding of pulsar physics. Any other possible processes that lead to instability to pulsar spin down energy could give timing residuals similar to our result, and may be in agreement with the observations as well as ours.

We thank the members at PKU pulsar group for helpful discussions. This work is supported by NSFC (10833003, 10935001, 10973002) and the National Basic Research Program of China under Grant No 2009CB824800.

\section{REFERENCES}

[1] Xu R X and Qiao G J 2001 Astrophys.J.Lett. 561 L85

[2] Yue Y L, Xu R X and Zhu W W 2007 Adv.SpaceRes. 401491

[3] Contopoulos I and Spitkovsky A 2006 Astrophys.J. 6431139

[4] Kramer M, et al. 2006 Science 312549

[5] Groth E J 1975 Astrophys.J.Suppl. 29431

[6] Stairs I H, Lyne A G and Shemar S 2000 Nature 406484

[7] Bailes M, Lyne A G and Shemar S L 1993 Astron.Soc.PacificConf.Ser. 3619

[8] Cordes J M 1993 Astr.Soc.PacificConf.Ser. 3643

[9] Jenet F A, Hobbs G B, Lee K J and Manchester R N 2005 Astrophys.J. 625123

[10] D'Alessandro F et al. 1995 Mon.Not.R.Astron.Soc. 2771033

[11] Hobbs G, Lyne A G and Kramer M 2006 Chin.J.Astron.Astrophys. 6169

[12] Chukwude A E 2007 Chin.J.Astron.Astrophys. 7521

[13] Hobbs G, Lyne A G and Kramer M 2010 Mon.Not.R.Astron.Soc. 4021027

[14] Stinebring D R et al. 2000 Astrophys.J. 539300 
[15] Na X S et al. 2009 arXiv : 0910.1453v1 [astro - ph.IM]

[16] Lyne A G et al. 2010 Science 329408

[17] Ryan M S et al. 2010 arXiv : 1010.4794v1[astro - ph.SR]

[18] Cordes J M and Downs G S 1985 Astrophys.J.Suppl. 59343

This preprint was prepared with the AAS LATEX macros v5.0. 

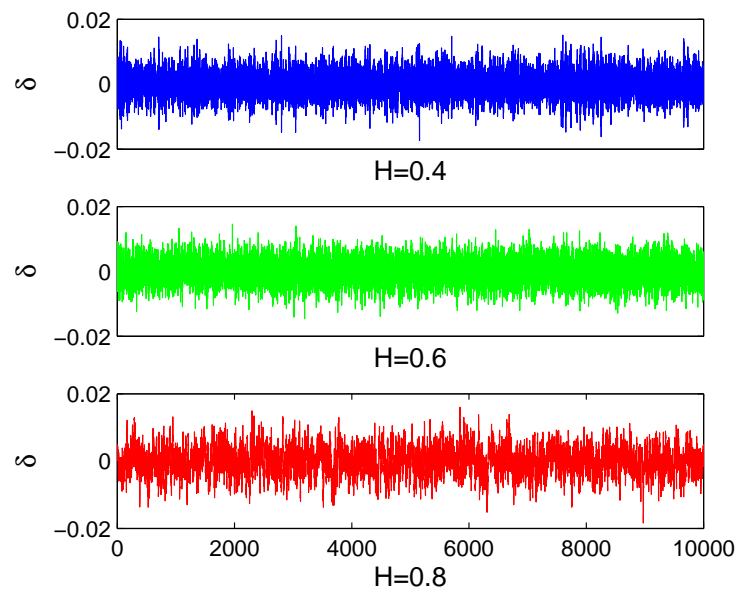

Fig. 1. - The data used to simulate the fluctuation of relativistic particles flux. The first set of data has more short period component, the second set is approximate white noise, the third set has more long period component. Here $H$ is the Hurst parameter.
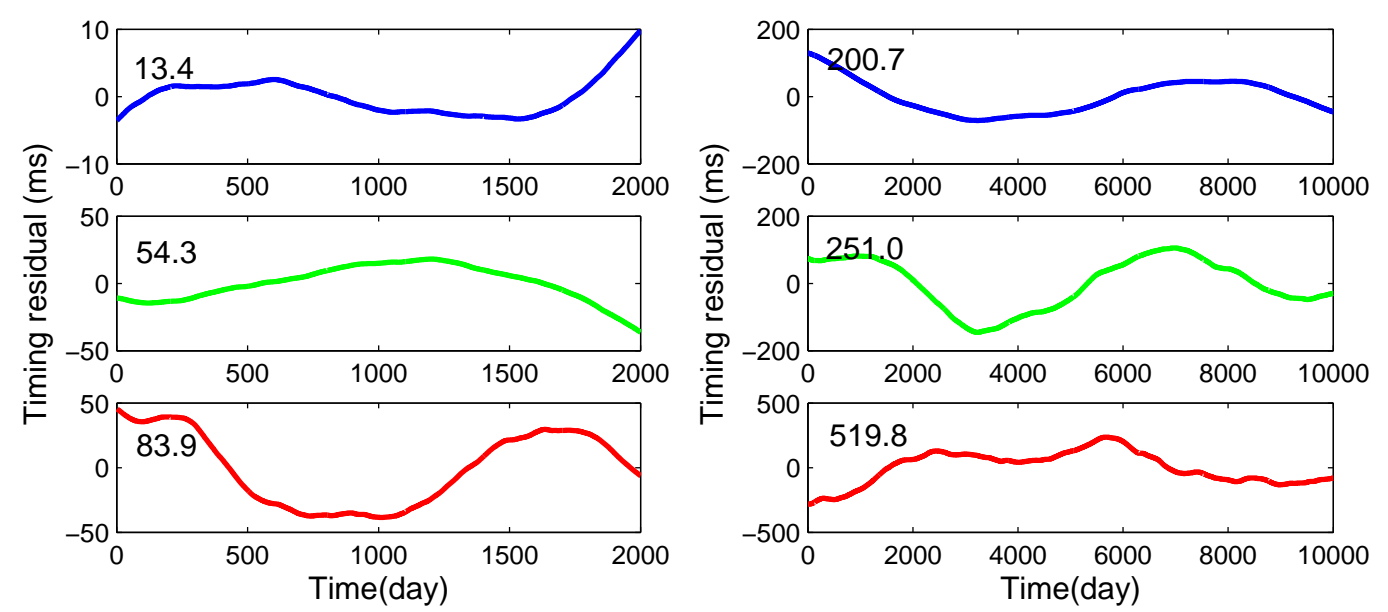

Fig. 2.- Curves of timing noise produced from the fluctuation data shown in Fig. 1. The Hurst parameters in the upper, middle, and bottom panels are $H=0.4,0.6,0.8$. The first 2000 points and the whole 10000 points are used in the left and right panels, respectively. We take $\left(n+1+\delta_{0}\right) P_{0}=$ $0.1 \mathrm{~s}$ and $\dot{P}_{0}=1 \times 10^{-14}$. The time spans are about 5.5 and $27 \mathrm{yr}$, and the label on the left of each panel provides the range from the minimum to the maximum residual (ms). The features of curve profile and noise rang are consistent with the observations. 